\title{
A proposed mechanism influencing structural patterns in X-linked retinoschisis and stellate nonhereditary idiopathic foveomacular retinoschisis
}

\author{
Serena Fragiotta ${ }^{1,2,3,4} \cdot$ Belinda C. S. Leong ${ }^{1,2,3} \cdot$ Talia R. Kaden $^{1,2,3,5} \cdot$ Sherry J. Bass $^{6} \cdot$ Jerome Sherman $^{6}$. \\ Lawrence A. Yannuzzi ${ }^{1,2,3,5} \cdot$ K. Bailey Freund ${ }^{1,2,3,5}$
}

Received: 04 September 2018 / Accepted: 11 September 2018 / Published online: 5 December 2018

(c) The Royal College of Ophthalmologists 2018

\begin{abstract}
Objective To explore the structural differences between X-linked retinoschisis (XLR) and stellate nonhereditary idiopathic foveomacular retinoschisis (SNIFR) using swept-source optical coherence tomography angiography (SS-OCTA).

Methods A case series of two patients, a 9-year-old male with XLR and a 58-year-old woman with SNIFR were imaged with swept-source optical coherence tomography angiography (SS-OCTA; PLEX Elite 900, Carl Zeiss Meditec, Inc, Dublin, CA). Automated segmentation was manually adjusted to include the areas of retinoschisis within en face flow and structural slabs. The flow data were binarized using ImageJ 1.51s (Wayne Rasband, National Institutes of Health, USA, http://imagej. nih.gov.ij) and superimposed onto the structural slab.

Results In the eye with XLR, OCTA flow data superimposed on the structural slab demonstrated flow signal within numerous bridging structures connecting the inner and outer plexiform layers containing the intermediate (ICP) and deep (DCP) capillary plexuses. In contrast, the same technique applied to the eye with SNIFR demonstrated an absence of flow signal in the cystic retinal spaces within Henle's fiber layer.

Conclusions The vascular pattern of bridging vessels between the ICP and DCP is closely related to the structural "retinoschisis" pattern of XLR and appears to be structurally different from that seen in SNIFR. Moreover, the connecting vessels appear to be highly represented and regularly distributed, thereby supporting a serial arrangement of the retinal capillary plexuses within the perifoveal macula.
\end{abstract}

K. Bailey Freund

kbfnyf@aol.com

1 Vitreous Retina Macula Consultants of New York, New York, NY, USA

2 LuEsther T. Mertz Retinal Research Center, Manhattan Eye, Ear and Throat Hospital, New York, NY, USA

3 Department of Ophthalmology, New York University School of Medicine, New York, NY, USA

4 Department of Medico-Surgical Science and Biotechnologies, Sapienza University of Rome, Rome, Italy

5 Department of Ophthalmology, Manhattan Eye, Ear and Throat Hospital, New York, NY, USA

6 State College of Optometry, The State University of New York (SUNY), New York, NY, USA

\section{Introduction}

$\mathrm{X}$-linked retinoschisis (XLR) is an inherited retinal disease caused by a mutation in the gene RS1 (Xp22.13) encoding retinoschisin [1]. Retinoschisis in XLR occurs primarily in the inner nuclear layer (INL) [1]. Stellate nonhereditary idiopathic foveomacular retinoschisis (SNIFR) is more common in women who lack RS1 mutations and other known predisposing factors for retinoschisis [2]. The stellate clinical appearance in SNIFR relates to splitting in Henle's fiber layer (HFL) at the posterior border of the outer plexiform layer (OPL) [2]. Herein we use structural and angiographic sweptsource optical coherence tomography to demonstrate key differences between these two types of retinoschisis.

\section{Case 1}

A 9-year-old male with a history of XLR was referred for evaluation. His family history included a brother and 


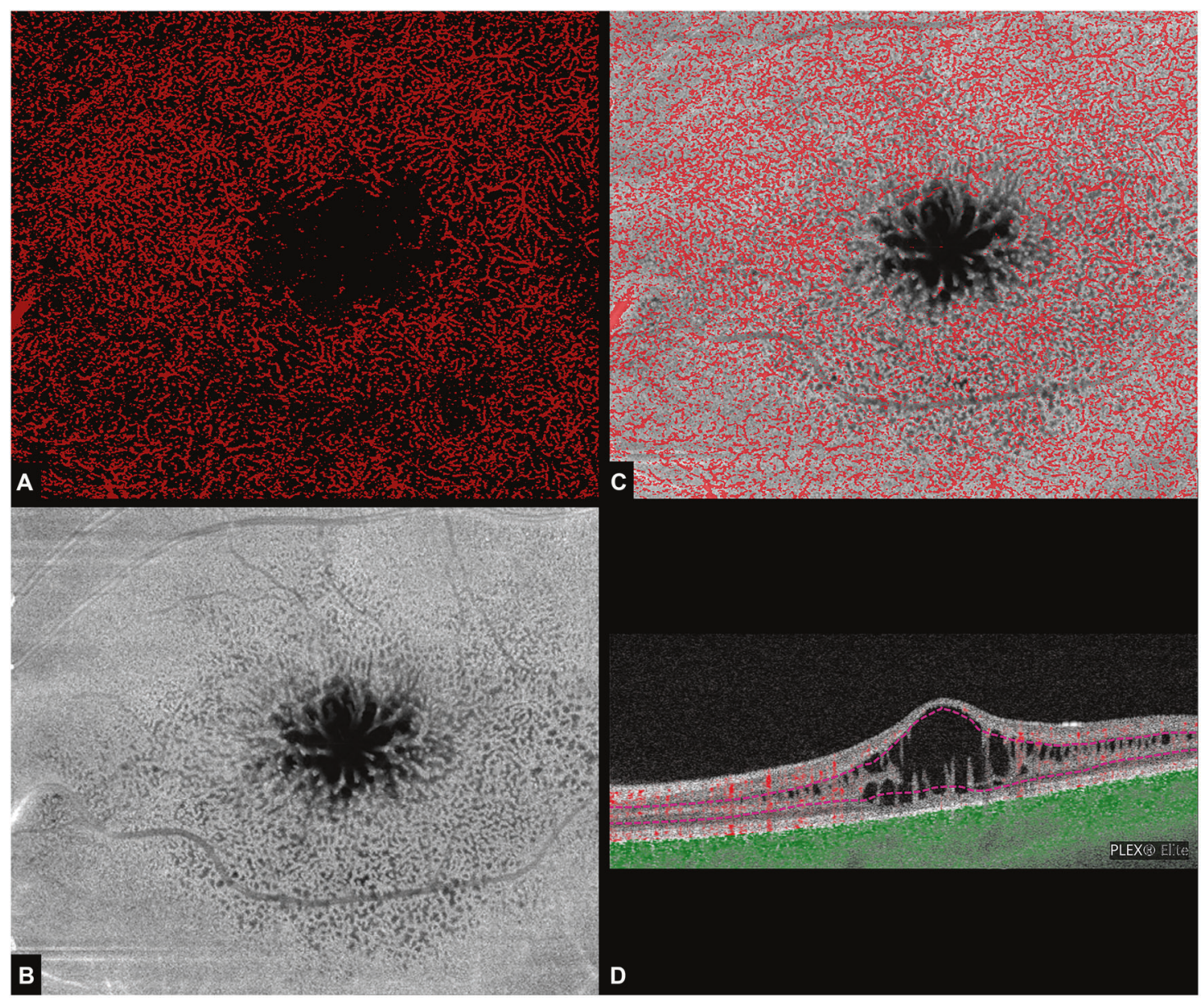

Fig. 1 Optical coherence tomography angiography (OCTA) in Xlinked retinoschisis. a Binarized en face OCTA with segmentation of the schitic area and projection removal. b En face structural slab. c OCTA flow signal (red) superimposed on the structural slab shows that flow signal representing bridging vessels between the intermediate and deep capillary plexuses is present in the large majority of connecting

maternal cousin with XLR. The patient reported progressive vision loss despite a recent trial of twice daily topical dorzolamide $2.0 \%$ and ketorolac $0.5 \%$ in both eyes. On examination, visual acuity was $20 / 100$ and $20 / 80$ in the right and left eyes, respectively. Ophthalmoscopy showed macular retinoschisis in both eyes. Multimodal imaging was undertaken, including $6 \times 6 \mathrm{~mm}$ swept-source optical coherence tomography angiography (SS-OCTA; PLEX Elite 900, Carl Zeiss Meditec, Inc, Dublin, CA). Segmentation of the retinoschisis in the left eye was performed on en face flow and structural slabs. The flow data were then binarized using ImageJ 1.51s (Wayne Rasband, National Institutes of Health, USA, http://imagej.nih.gov.ij), as demonstrated in Fig. 1. By superimposing the binarized en face flow image (Fig. 1a, red) onto the structural slab (Fig. 1b), it was noted that multiple discrete areas of flow signal representing bridging vessels between the intermediate capillary plexus (ICP) and deep capillary plexus tissue within the inner nuclear layer. Black spaces correspond to intraretinal cysts. d OCT B-scan with flow overlay shows segmentation lines (dashed magenta lines) used for the en face projections (a and $\mathbf{b}$ ) and confirms correspondence of flow signal with the tissue bridging the schisis

(DCP) accompanied the large majority of connecting structures (Fig. 1c). Cross-sectional imaging also demonstrated flow signal within this bridging tissue (Fig. 1d).

\section{Case 2}

A 58-year-old woman was recently diagnosed with SNIFR. There was no family history of retinal disease. On examination, visual acuity was $20 / 20$ in both eyes. Ophthalmoscopy showed a stellate pattern of macular retinoschisis in both eyes. SS-OCTA was performed using the same $6 \times 6 \mathrm{~mm}$ acquisition mode and subsequent image analysis as in case 1 (Fig. 2a-c). Based on the retinoschisis location, segmentation of HFL was employed (Fig. 2d). Similar superimposition of the en face image demonstrated the absence of flow signal within the retinal tissue separating the cystic spaces. 


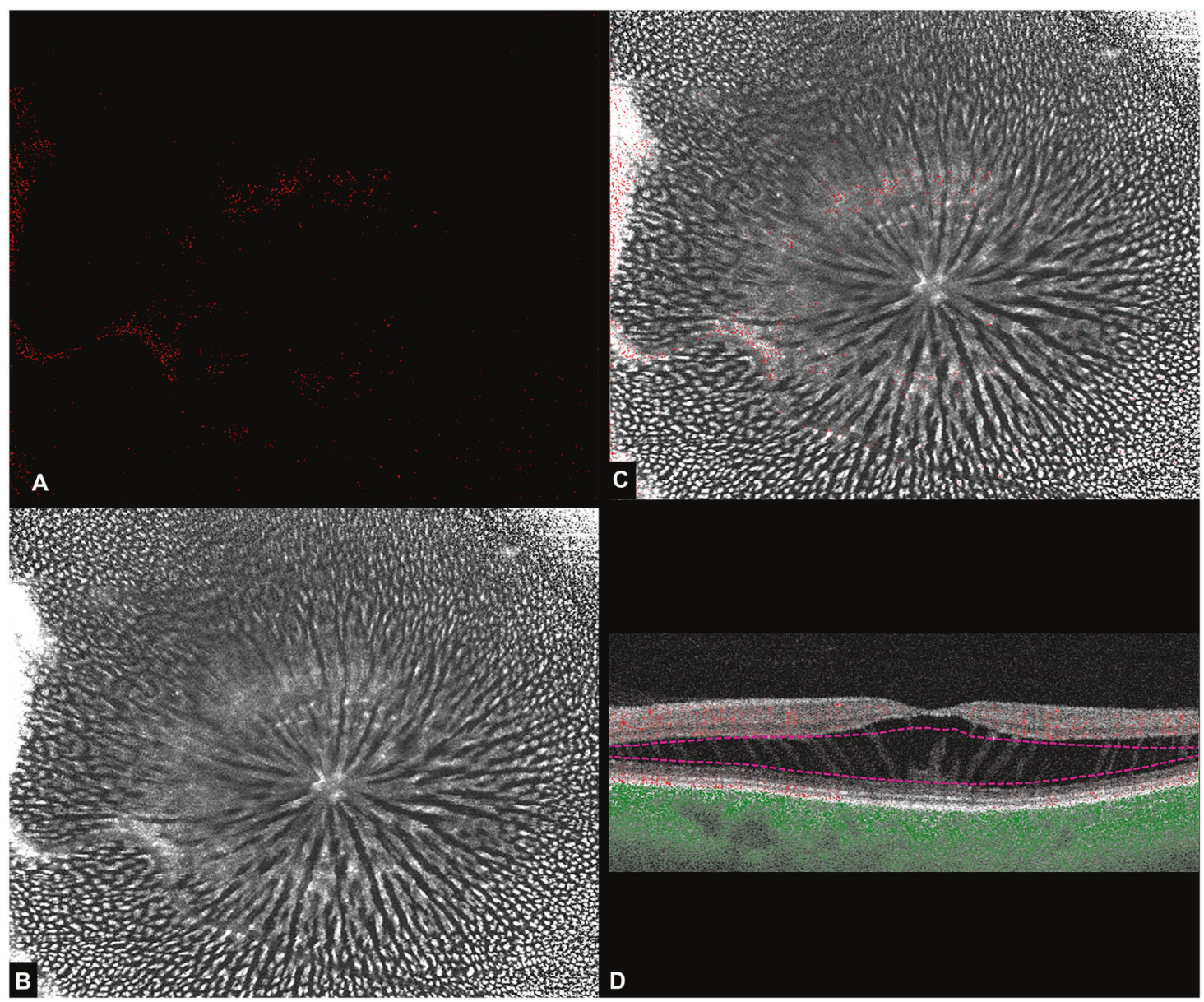

Fig. 2 Optical coherence tomography angiography (OCTA) in stellate nonhereditary idiopathic foveomacular retinoschisis. a Binarized en face OCTA of the schitic area with projection removal. b En face structural slab. c OCTA flow signal (red) superimposed on the structural slab demonstrates the absence of flow signal within retinal tissue

\section{Discussion}

While both XLR and SNIFR have radial patterns of cysts within the retina, the patterns themselves are quite different. In XLR, the retinoschisis primarily involves the INL which contains the bridging vessels between the ICP and DCP that together comprise the deep vascular complex [3, 4]. The bridging structures within the retinoschisis appeared to align with retinal blood vessels on both en face and crosssectional OCTA views (Fig. 1). We postulate that the structural support provided by these connecting vessels influences the pattern of intraretinal cavities and prevents a wider separation between retinal layers, except at the foveal avascular zone where Müller cells are plentiful but vessels are absent. This observation is confirmed by prior histologic and OCT studies that demonstrate that the bridging structures are composed of glial material that has features consistent with Müller cells surrounding blood vessels with thickened walls $[1,5,6]$.

separating cystic spaces within Henle's fiber layer. d OCT B-scan with flow overlay shows segmentation lines (dashed magenta lines) used for the en face projections (a and $\mathbf{b}$ ) and confirms an absence of flow signal within the tissue bridging the schisis

The perifoveal pattern of INL retinoschisis in XLR, characterized by multiple small regularly spaced hyperreflective spaces seen with OCT B-scans, resembles that of cystoid macular edema occurring in retinitis pigmentosa where intraretinal cavities are most commonly found in the INL [7]. A similar pattern of INL cavitation called "microcystic macular edema" occurs in a range of optic neuropathies including those associated with multiple sclerosis, neuromyelitis optica, and glaucoma [8-12]. While the underlying cause of these structural changes may vary with each associated disease, the numerous regularly spaced vessels bridging the INL between the ICP and DCP are likely to influence the anatomic pattern seen with all. In addition, shearing stress or rupture of bridging vessels may help explain certain unique patterns of fluorescein angiographic leakage and intrarenal hemorrhage occurring in these and other retinal disease affecting INL structure.

In contrast, the location and pattern of the retinoschisis observed in SNIFR is consistent with pillars of avascular 
retinal tissue comprised of Müller fibers and photoreceptor axons [4, 13]. The absence of vascular flow signal in the bridging tissue involving HFL was readily apparent in our patient (Fig. 2). This typical stellate pattern characterized by radially oriented spokes of tissue may be a result of limited resistance offered by involved tissue lacking the structural support provided by local blood vessels.

The sheer number of regularly spaced vascular connections found between the ICP and DCP in XLRS appears to support a predominantly serial arrangement for the retina capillary plexuses within the perifoveal macula [14-17]. Our findings would be difficult to reconcile with recently proposed parallel or "hammock" arrangement of macular vascular flow in which vessels connecting the DCP and ICP are described as infrequent or absent [4, 18]. Proponents of a parallel arrangement argue that distinct neurovascular units at the IPL/ICP and OPL/DCP require independent arteriolar supply and venous drainage [4]. In the "serial" model, there are numerous bridging vessels between the vascular plexuses with venous drainage originating predominantly in the DVC [14-16, 19, 20]. Macular venous drainage through the DVC was recently supported by the observation that, when studied with OCTA, all collateral vessels formed in the macula following branch retinal vein occlusion were found to course through the DVC [14].

In summary, we describe the novel observation that the numerous small vessels bridging the INL between the ICP and DCP likely play an important role in driving the pattern of INL "retinoschisis" occurring in a range of neurodegenerative retinal diseases. Moreover, the high number and regular distribution of these connection vessels appears to support a series arrangement of the retinal capillary plexuses within the macula.

\section{Summary}

\section{What was known before:}

- X-linked retinoschisis (XLR) and stellate nonhereditary idiopathic foveomacular retinoschisis (SNIFRS) are two forms of retinoschisis involving different retinal layers and each having a different structural pattern.

- In the "serial" model of retinal capillary plexuses, there exist numerous bridging vessels between the vascular plexuses, with venous drainage originating predominantly in the deep vascular complex.

\section{What this study adds:}

- While retinoschisis in SNIFR involves an avascular Henle's fiber layer, the inner nuclear layer (INL) retinoschisis of XLR includes tissue containing numerous vessels bridging the intermediate and deep capillary plexuses which likely influence the structural pattern and degree of separation occurring at this level.

- The evidence of regularly spaced bridging vessels between the capillary plexuses bordering the INL seems to add support for a "serial" arrangement of the perifoveal retinal vascular flow.

\section{Acknowledgements}

Funding This work was supported by The Macula Foundation, Inc., New York, NY. SF was supported by the H2CU-Honors Center of Italian Universities.

\section{Compliance with ethical standards}

Conflict of interest $\mathrm{KBF}$ is a consultant to Genentech, Allergan, Optos, Optovue, Zeiss, Heidelberg Engineering, and Novartis. He receives research funding from Genentech/Roche. JS is a consultant for Topcon, Diopsys, and INNOVA. The remaining authors declare that they have no conflict of interest.

\section{References}

1. Orès R, Mohand-Said S, Dhaenens C-M, Antonio A, Zeitz C, Augstburger E, et al. Phenotypic characteristics of a French cohort of patients with X-linked retinoschisis. Ophthalmology. 2018. https://doi.org/10.1016/j.ophtha.2018.03.057.

2. Ober MD, Freund KB, Shah M, Ahmed S, Mahmoud TH, Aaberg $\mathrm{TM}$, et al. Stellate nonhereditary idiopathic foveomacular retinoschisis. Ophthalmology. 2014;121:1406-13.

3. Snodderly DM, Weinhaus RS, Choi JC. Neural-vascular relationships in central retina of macaque monkeys (Macaca fascicularis). J Neurosci. 1992;12:1169-93.

4. Campbell JP, Zhang M, Hwang TS, Bailey ST, Wilson DJ, Jia Y, et al. Detailed vascular anatomy of the human retina by projection-resolved optical coherence tomography angiography. Sci. Rep. 2017;7:42201.

5. Lee EJ, Kim T-W, Kim M, Choi YJ. Peripapillary retinoschisis in glaucomatous eyes. PLoS ONE. 2014;9:e90129.

6. Condon GP, Brownstein S, Wang NS, Kearns JAF, Ewing CC. Congenital hereditary (juvenile $\mathrm{X}$-linked) retinoschisis: histopathologic and ultrastructural findings in three eyes. Arch Ophthalmol. 1986;104:576-83.

7. Makiyama Y, Oishi A, Otani A, Ogino K, Nakagawa S, Kurimoto $\mathrm{M}$, et al. Prevalence and spatial distribution of cystoid spaces in retinitis pigmentosa. Retina. 2014;34:981-8. http://content.wkhea lth.com/linkback/openurl?sid=WKPTLP:landingpage \&an $=$ 00006982-201405000-00020

8. Hasegawa T, Akagi T, Yoshikawa M, Suda K, Yamada H, Kimura $\mathrm{Y}$, et al. Microcystic inner nuclear layer changes and retinal nerve fiber layer defects in eyes with glaucoma. PLoS ONE. 2015;10:e0130175.

9. Fortune B, Ma KN, Gardiner SK, Demirel S, Mansberger SL. Peripapillary retinoschisis in glaucoma: association with progression and OCT signs of Müller cell involvement. Investig Ophthalmol Vis Sci. 2018;59:2818-27.

10. Gelfand JM, Nolan R, Schwartz DM, Graves J, Green AJ. Microcystic macular oedema in multiple sclerosis is associated with disease severity. Brain. 2012;135:1786-93. 
11. Abegg M, Dysli M, Wolf S, Kowal J, Dufour P, Zinkernagel M. Microcystic macular edema. Ophthalmology. 2014;121:142-9.

12. Brazerol J, Iliev ME, Höhn R, Fränkl S, Grabe H, Abegg M. Retrograde maculopathy in patients with glaucoma. J Glaucoma. 2017; 26:423-9.

13. Mrejen S, Gallego-Pinazo R, Freund KB, Paques M. Recognition of henle's fiber layer on OCT images. Ophthalmology. 2013;120: e32-3.e1.

14. Freund K, Sarraf D, Leong BCS, Garrity S, Vupparaboina KK, Dansingani KK. Association of optical coherence tomography angiography of collaterals in retinal vein occlusion with major venous outflow through the deep vascular complex. JAMA Ophthalmol. 2018. https://doi.org/10.1001/jamaophthalmol.2018.3586.

15. Garrity ST, Paques M, Gaudric A, Freund KB, Sarraf D. Considerations in the understanding of venous outflow in the retinal capillary plexus. Retina. 2017;37:1809-12.

16. Garrity ST, Iafe NA, Phasukkijwatana N, Chen X, Sarraf D. Quantitative analysis of three distinct retinal capillary plexuses in healthy eyes using optical coherence tomography angiography. Invest Ophthalmol Vis Sci. 2017;58:5548-55.

17. Fouquet S, Vacca O, Sennlaub F, Paques M. The 3D retinal capillary circulation in pigs reveals a predominant serial organization. Investig Ophthalmol Vis Sci. 2017;58:5754-63

18. Nesper PL, Fawzi AA. Human parafoveal capillary vascular anatomy and connectivity revealed by optical coherence tomography angiography. Invest Ophthalmol Vis Sci. 2018;59: 3858-67.

19. Ghasemi Falavarjani K, Phasukkijwatana N, Freund KB, Cunningham ET, Kalevar A, McDonald HR, et al. En face optical coherence tomography analysis to assess the spectrum of perivenular ischemia and paracentral acute middle maculopathy in retinal vein occlusion. Am J Ophthalmol. 2017;177:131-8.

20. Garrity ST, Tseng VL, Sarraf D. Paracentral acute middle maculopathy in a perivenular fern-like distribution with en-face optical coherence tomography. Retin Cases Brief Rep. 2017;12 Suppl 1: S25-S28. 